\title{
Design Fuzzy Controller Based on Function Self-tuning*
}

\author{
Hui Wang, Bobo Zhang, Lijun Yu, Zuoyu Guan \\ College of Automation, Harbin Engineering University, Harbin, China \\ wangh@hrbeu.edu.cn
}

\begin{abstract}
Fuzzy Control is one of important and effective way in intelligent control, with its unique fuzzy theory, summed control rules directly from the experiences of control, opened up new avenues for the time-lag systems. In order to make the fuzzy control system can maintain a rapid and efficient control, the introduction of the adaptive method in fuzzy control system, composed of adaptive fuzzy control system. Adaptive control is proposed scale factor and to quantify the use of factor function to achieve self-adjustment. Through MATLAB simulation, function self-tuning controller of the system transition process shorter time, rapid deformation, and improve the accuracy.

Index Terms - Fuzzy Control, Adaptive, Function self - tuning, Accuracy
\end{abstract}

\section{I . Introduction}

The time-lag system is widespread in the industrial production system. Due to its pure delay effect, making the amount of control can't be reflected in the controlled object, the resulting control system overshoot, steady-state error and stability control performance is significantly deteriorated ${ }^{[1-3]}$. Fuzzy control as an important and effective method of intelligent control, fuzzy theory, with its unique summed control rules directly from the experience of control, system control method for the time-lag has opened up new avenues ${ }^{[4]}$. The core of the fuzzy control is fuzzy controller design and it's directly related to the control effects of good or bad. Control rules, membership function, scale factor and quantitative factor select appropriate or not, affect the control accuracy and dynamic performance meets the requirements of practical engineering or not ${ }^{[5,6]}$.

In order to improve the basic fuzzy controller static performance and dynamic performance can't achieve optimal at the same time ${ }^{[7]}$, improve its control through the following channels: modify the fuzzy control table; tune the system's quantization factor and scaling factor online; modify the set of words in each language variable fuzzy sets. But there were still various problems, for example, which parameters as the adjustment object, how to coordinate the relationship between the various parameters, as well as the design of complex, inconvenient timely adjustment and so on.

This article from the linear quadratic optimal control used variable matrix to better adapt to a variety of special circumstances. The fuzzy controller is proposed the use of self-tuning function, the relative parameters fuzzy controller obviously been optimized, while the relative existing parameter self-tuning fuzzy controller is designed to be simple, easy to modify, can modify the function to improve the control effect of the different requirements at any time.

\section{General Parameters Fuzzy Logic Controller}

The error and error rate of change is selected as the basic fuzzy controller's input signal, combined with its fuzzy knowledge base fuzzy inference and defuzzification output as the amount of control the controlled object ${ }^{[8]}$.

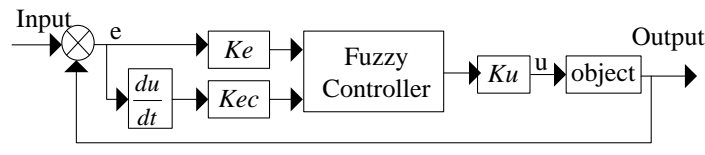

Fig. 1 Ordinary Fuzzy Controller

When $e$ and $e c$ into the fuzzy controller as input signal and the fuzzy controller output signal as a control signal with the scale factor and the quantization factor adjustment, the scale factor and the quantization factor is fixed in the basic fuzzy control, but the impacts of the scale factor and quantify factors on the control effect are the opposite, which can't at the same time get a better dynamic performance and steady-state performance, so compromise into account only select the better $K_{\mathrm{e}}, K_{\mathrm{ec}}$ and $K_{\mathrm{u}}{ }^{[9]}$.

In order to solve the above can't select the optimum $K_{\mathrm{e}}$, $K_{\text {ec }}$ and $K_{\mathrm{u}}$, experts had designed self-tuning online fuzzy controller. Professor $\mathrm{Li}$ Hong xing brought the variable universe ideological to make parameters self-adjusting, and put forward the concept of elastic factor ${ }^{[9]}$, its essence is the basic domain of the input and output with the control requirements according to certain criteria for the appropriate time scale change, the definition also changes in the basic domain. Fig. 2 shows, by designing parameters to adjust, has resolved conflicting relationship between the quantization factor and the scale factor, however, both of the adjustment required by the membership function selecting and determining the mathematical relationship, so increasing the controller design difficulty, while increasing the control parameters need to design a controller which increases the complexity, the mathematical relationship proposed by Professor Li Hongxing hard to calculate and the function is a complex relationship, so it's not suitable for re-adjustment in engineering practice.

\footnotetext{
${ }^{*}$ This paper is funded by the central university basic research operations special fund project (HEUCF041315), and the International Exchange Program of Harbin Engineering University for Innovation-oriented Talents Cultivation.
} 


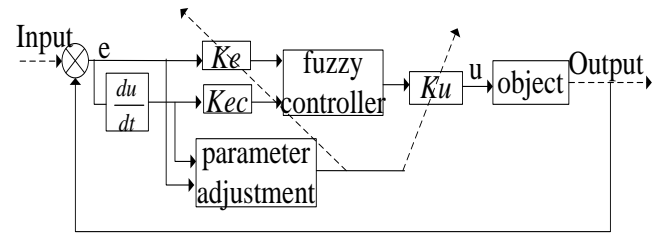

Fig. 2 Adaptive Fuzzy Controller

\section{Function - based Parameter Self - tuning Controller Design}

The basic idea of the adaptive fuzzy control is to adjust the control rule based on error change to adapt the change. The fuzzy control start from fuzzifying the exact value to fuzzy reasoning, fuzzy decision to clarity the whole proceeding by difference function, but the output of fuzzy control is to approach the real response function, and the difference will meet the demand when the approach is enough. The essence of adaptive fuzzy control is to change the difference at any time to get the desired control effect.

From the monotonic definition of control effect, that the monotonic of difference function and control rule is equal, can be drawn, so the non-controdiction of control rule can be ensured with the changing domain ${ }^{[10]}$, which provide powerful theoretical basis for this paper.

\section{A. Function Self-tuning Fuzzy Controller}

A simple method designed in this paper can get selfadjusting of $K_{\mathrm{e}}, K_{\mathrm{ec}}$ and $K_{\mathrm{u}}$ to improve the control effect, but also can ensure the practical engineering control demand. The method is: transform the linear change with proportional relationship to linear change with non-proportional relationship, the fixed value be replaced by function, and selecting the appropriate function relationship by the principle of selecting $K_{\mathrm{e}}, K_{\mathrm{ec}}$ and $K_{\mathrm{u}}$ can get the self-adjusting of scale factor and quantization factor. The figure is following.

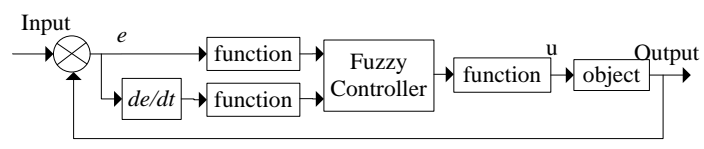

Fig. 3 Function self-tuning controller

Let $e(e c)$ basic domain be [-e,e]([-ec,ec]), fuzzy domain be $[-n,-n+1, \ldots, 0, \ldots, n-1, n]$, then the precise amount of fuzzy quantification factor :

$$
K_{e}=\frac{n}{e}\left(K_{e c}=\frac{n}{e c}\right)
$$

Let $u$ basic domain be [-u,u], fuzzy domain be [-m,$\mathrm{m}+1, \ldots, 0, \ldots, \mathrm{m}-1, \mathrm{~m}]$, Fuzzy amount of precision scale factor :

$$
K_{u}=\frac{u}{m}
$$

From the above two formulas, it can be seen that, the error and the error change rate change linearly with the control amount. But it can't meet the practical demand getting $K_{\mathrm{e}}, K_{\mathrm{ec}}$ and $K_{\mathrm{u}}$ in this way, so large number of simulation and expertise are needed, which increases the control difficulties.

Variable universe adaptive fuzzy controller changes the input and output universe by using elastic factor to change the control rule. Assume that the initial domain is $[-\mathrm{X}, \mathrm{X}]$, the elastic factor is $\left(x_{\mathrm{i}}\right)$, and a new domain $\left(\left[-\alpha\left(x_{\mathrm{i}}\right) \mathrm{X}, \alpha\left(x_{\mathrm{i}}\right) \mathrm{X}\right]\right)$ can be got with xi changing, then change the way of inserting numbers to make the output function of controller approach the real response function. This idea is used in this paper to control the system. Set variable is $\mathrm{X}(t)$, the domain is $[-y, y]$ and the variable function is $f(x)$, so they have the following relationship.

$$
f(x)=\frac{y}{x(t)}
$$

The scale factor and quantization factor changes with $x(t)$ changing, also the changes of domain is the response of $f(x)$ changing, which can get self-adjusting parameters. The definition of control rule monotonic shows that the contradiction of domain changing and control rule can be avoided as long as the function monotonic is guaranteed.

\section{B. Scale Factor and Quantitative Factors on the Control Effects}

According to equation (1), it can be seen that, the basic domain of the variables [-e,e] will gradually decrease with $K_{\mathrm{e}}$ increasing, the control effect will enhances to increase the system rate, which can make ec into saturation rapidly, so the oscillation generates. While the basic domain of the variables [-e,e] will gradually increase with $K_{\mathrm{e}}$ decreasing, the control effect of error will weaken, which will decrease the control sensitivity of error. Meanwhile the other conclusion can be got that, the larger $K_{\mathrm{ec}}$ is, the increasing rate will be smaller, the process will be longer and the system response will be slower. The smaller $K_{\mathrm{ec}}$ is, the system increasing rate will be larger, which may lead to excessive overshoot and shook, so that the system will be unstable ${ }^{[11]}$.

$K_{\mathrm{u}}$ is directly associated with the control amount, the effect on the fuzzy controller is more direct than $K_{\mathrm{e}}$ and $K_{\mathrm{ec}}{ }^{[12]}$. From the equation (2), it can be seen that, the overshoot of the system increases with increasing $K_{\mathrm{u}}$, dynamic process will speed up, and bring about larger overshoot or system instability; on the contrary, the overshoot of the system decreases with decreasing $K_{\mathrm{u}}$, the dynamic process will be slower and the adjusting time will be longer with poor steadystate accuracy.

\section{Strategy of Self-adjusting Fuzzy Controller Function Selection}

In the initial stages of the control process, $e$ and $e c$ are bigger, so smaller $K_{\mathrm{e}}$ and $K_{\mathrm{ec}}$ should be selected to reduce the resolution of the input $e$ and $e c$. According to (3), a smaller function value should be selected with large error and error change rate, select to ensure a larger domain. On the contrary, a larger $K_{\mathrm{u}}$ is selected to increases the control amount, speed up the response to get a larger function value, so the coarse adjustment can be got. Thus, it is possible to design the 
function respectively to get demanded scale factor and quantization factor $^{[13]}$.

With fuzzy control process, the error and error change rate gradually decreases, and the scale factor and the quantization factors also change, so the control effect can be optimized. When $e$ and $e c$ become smaller, $K_{\mathrm{e}}$ and $K_{\mathrm{ec}}$ should be larger to improve the resolution of the input $e$ and $e c^{[14]}$. According to the (3), it can be seen that, select a larger function value, enlarge the domain, select the smaller $K_{\mathrm{u}}$, reduce the change of control amount of control, constrain the overshoot increase of the system, select a smaller function value, amplify the control about the control object, so that the system can be stable as soon as possible to fine-tune the system.

According to the adjusting strategy of scale factor and quantization factor and their relationship between each other, and considering the monotonic change, the alike function of $K_{\mathrm{e}} / K_{\text {ec }}$ can be chosen as shown in Fig. $4 K_{\mathrm{e}} / K_{\mathrm{ec}}(\mathrm{b})$ curve, and the alike function of $K_{\mathrm{u}}$ can be chosen as shown in Fig. $4 K_{\mathrm{u}}(\mathrm{a})$ curve. So the fuzzy adaptation can be achieved.

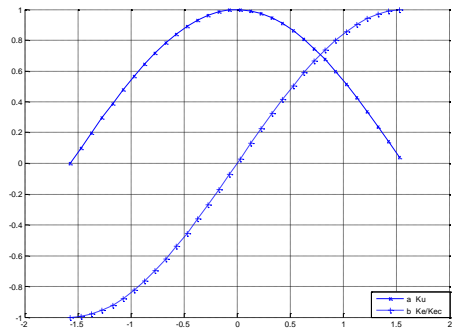

Fig. 4 Function's Figure of $K_{\mathrm{e}}, K_{\mathrm{ec}}$ and $K_{\mathrm{u}}$

Considering the multi-input and single-output fuzzy control model avoiding the loss of generality, to ensure the practicality of the selected function, several conditions are needed of the functions:

(1) As an alternative function of the quantization factor, one would be: when $|x|=0, f(x)=1$, which can narrow the domain to enhance the control sensitivity; on the contrary, the scaling factor would be: $f(x)=0$, which can guarantee the zero output of the control amount.

(2) To fully guarantee the monotonicity of $f(x)$ in [-E,0] and $[0, \mathrm{E}]$ to ensure the consistency of the changes in function and fuzzy rules change to avoid conflicts .

(3) If the selected output domain is symmetrical, the alternative function should be an odd function.

\section{Determination of Coefficients}

Initial fuzzy controller design, it is necessary to determine the scope of the universe, but the universe is generally not easy to determine and estimated based on actual experiences. However, whether the domain of the range input the input range affected the control effects. For example, when the input variables were not in the range of domain, controller lost control actions; when the domain was too large, the amount of input did not reflect the good control effects. Use self-tuning function, can be good to avoid adverse on the domain-wide inappropriate control effects.

Dual-input single-output fuzzy controller is an example.

First, the input should be carried out to $|x| \leq 1$, then through (4) to determine the coefficients of the function.

$$
k=\frac{\max E}{\max f(x)}
$$

Where $\max E$ is the maximum value of the domain, $\max f(x)$ is the max input or the max output of fuzzy control, $k$ is the coefficient of the function.

The variable does not go beyond the boundaries of the universe, so that you can resolve the fuzzy domain should not be selected distress of the domain chosen arbitrarily.

In order to achieve fine control of the control object, you can select the function value is less than or equal to 1 function, so that $|u| \leq 1$.

\section{Simulation}

Select the second-order lag element $G(s)=\frac{1}{20 s^{2}+15 s+1} e^{-12 s}$ as the controlled object to research.

The domain of $e$ and $e c$ are [-6,6], fuzzy domains both are $[-6,-5, \ldots, 0, \ldots, 5,6]$, the domain of $u$ is $[-10,10]$, the fuzzy domain is $[-10,-9,-8, \ldots, 0, \ldots, 8,9,10]$, in order to ensure that the input variables on domain change, $K_{\mathrm{e}}$ and $K_{\mathrm{ec}}$ select $\cos (x)$ function. First, input variables are converted to $[-1,1]$, at the same time, in order to able to make full use of the input variables to the control rule tables, the coefficients of the function are 6 obtained by the formula (4). Similarly, $K_{\mathrm{u}}$ select $\sin \left((\mathrm{pi} / 20)^{*} x\right)$ function. To join an integrator, in order to eliminate the error inherent in the fuzzy control. Control model as shown Fig. 5.

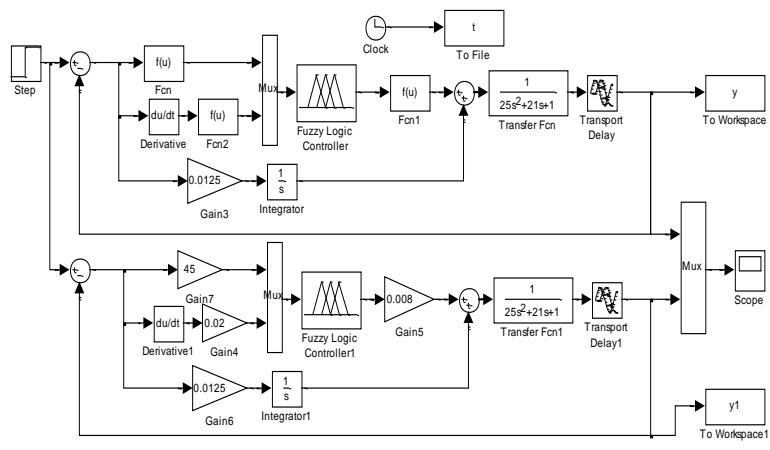

Fig. 5 Control Modle

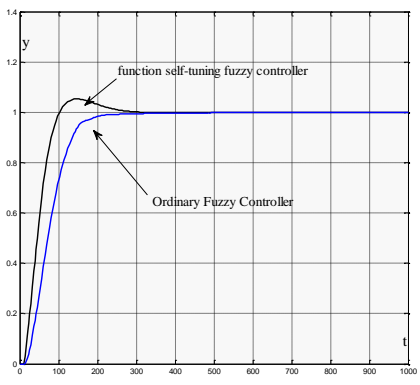

Fig. 6 Simulation Results 
Can be seen from the Fig. 6, the two control methods both have reached a stable control effect, but the effect of the use of self-adjusting fuzzy control was significantly better than the ordinary control the effect. The transition time of using self-adjusting function is obviously cut down, quickly reaches a steady state. Same time, by enlarge the vertical axis scale to carefully observe, the adjustment controller does not use the function, when the control achieves stable, there is also a slight error, but the use of the self-adjustment function, a smooth control is not errors.

\section{Conclusions}

The fuzzy control has a very wide range of application, so it has a very large theoretical value and practical significance to study a simple and practical fuzzy controller. The adjustment method mentioned in this paper is simple in application and function selection, thus it can greatly short the time to get the optimum scale factor and quantitative factors by repeated simulation with the satisfactory practical application. But without systems theory analysis to coordinate the functional relationship, an in-depth study is needed based on the basic theory.

\section{References}

[1] Jiaojiao Zhang, Jianjiao Ji, Jingxin Si, Jie Xu. The lag system control method. Science and Technology Innovation Herald, 2009, 23): 210, Chinese;

[2] Wenzhen Cai. The lag system control method. SCIENCE \& TECHNOLOGY INOFOMATION, 2008, (08): 223-225, Chinese;
[3] WEI Qiu-yue, WANG Wen-qing. Fuzzy Self-adaptive PI-Smith Control for Long Time-delay System. Automation \& Instrumentation, 2011,(4):27-30, Chinese;

[4] Ning Yao. Control Strategy and Simulation on Time Delay System. AUTOMATION PANORAMA, 2010(4):75-77, Chinese;

[5] Xiaoyan Yang. Design of Parameter Self-tuning Fuzzy Controller Based on Matlab. AUTOMATION PANORAMA, 2009(12):76-79, Chinese;

[6] Haigang Guo, Hongxing Li, Kai Hu. A Novel Variable Universe Adaptive Fuzzy Controller. Fuzzy Systems and Mathematics, 2011(12):1-9, Chinese;

[7] Wang Lei-lei, Wang Meng-xiao. Design and simulation of fuzzy IMCPID controller. Computer Engineering and Applications, 2008, 44(32): 220-222, Chinese;

[8] Wang Jia-yin, Liu Min, Li Hong-xing. Analysis of Difference Between Control Function and Interpolation Expression of SISO Fuzzy Controller. ACTA ELECTRONICA SINICA,2009,(2):424-428, Chinese;

[9] Meng Yanping, Tan Yanhua, Li Hongxing. Adaptive Fuzzy Systems and the Analysis of Approximation. Advances In Mathematics, 2012,(04):423-435, Chinese;

[10] Wang Zhixin. Intelligent Fuzzy Control Research on Some Problems. Intellectual Property,2009,9, Chinese;

[11] Long Zuqiang. Research on Some Important Issues of Fuzzy Controllers with variable Universe of Discourse. Central south university,2011, Chinese;

[12] Lu Yongkun. Research of design method for stablevariable universe fuzzy control system. TIANJIN UNIVERSITY,2010, Chinese;

[13] WangHui, GuoXiao, An Analysis of the Minimal Effective Detector Set Based on the Adjustable Fuzzy Matching Negative Selection Algorithm of Immune · IEEE ICISE 2010,965-968, Chinese;

[14] Wang Hui, Yu Lijun, Wang Kejun, Zhang Lijun, An adjustable fuzzy matching negative selection algorithm, CAAI Transactions on Intelligent Systems, 2011,(02):178-184,Chinese. 